\title{
Acceptance and Hope for Achievement (Aha) Syndrome As Key Resilience for Sustaining Community Based Programmes
}

\author{
Fyson H. Kasenga ${ }^{1 *}$ and Lettice Kasenga ${ }^{2}$ \\ ${ }^{1}$ Academic Editor \& Researcher, Adventist Health Services, Blantyre, Malawi. \\ ${ }^{2}$ Malawi Adventist University, Malamulo College of Health Sciences Campus, Makwasa, Malawi.
}

*Corresponding author: Fyson H. Kasenga, Academic Editor \& Researcher, Adventist Health Services, Blantyre, Malawi.

Received date: June 11, 2021; Accepted date: June 17, 2021; Published date: June 24, 2021

Citation: Fyson H. Kasenga and Lettice Kasenga (2021). Use of the frailty tools FI MDS and FI Rockwood to predict outcomes of inpatient rehabilitation after proximal hip fracture. J Clinical Research and Reports, 8(3); DOI:10.31579/2690-1919/180

Copyright: (C) 2021 Fyson H. Kasenga, This is an open access article distributed under the Creative Commons Attribution License, which permits unrestricted use, distribution, and reproduction in any medium, provided the original work is properly cited.

\begin{abstract}
They are an answer to transforming communities from poverty to prosperity. However, caution should be observed because it is not every community based programme that will have the potential to transform a community positively. It depends on the approaches selected and used to empower the communities. Having worked with various communities in diverse cultures ranging from Malawi, Kenya and Tanzania among others, what has been observed is that communities respond to different initiatives differently but the principle remains the same and therefore is a common factor. The bottom line behind community based programmes is to create what is called 'community resilience'. In this write up this means that communities are empowered to depend on themselves and live within their means economically, socially, culturally, spiritually as well as psychologically with minimal or no external support at all (Quirk, R, 2001). Borrowing a leaf from an international economic order as coined by the Alma Ata Declaration of 1978 where it said that 'the rich and developed nations have the mandate to support the poor and developing nations' should not be take for granted. Should the support from rich and developed nations to the underdeveloped ones continue to make no positive impact on the nation in question then it becomes pathological, hence unacceptable. As such, the support needs not to continue but rather be revisited or another alternative should be sorted out.
\end{abstract}

Keywords: community based programmes; emotional; psychological; fresh potato crisps; pork sausages; AHA; diverse cultures; Malawi

\subsection{Background}

Why community based programmes? They are an answer to transforming communities from poverty to prosperity. However, caution should be observed because it is not every community based programme that will have the potential to transform a community positively. It depends on the approaches selected and used to empower the communities. Having worked with various communities in diverse cultures ranging from Malawi, Kenya and Tanzania among others, what has been observed is that communities respond to different initiatives differently but the principle remains the same and therefore is a common factor. The bottom line behind community based programmes is to create what is called 'community resilience'. In this write up this means that communities are empowered to depend on themselves and live within their means economically, socially, culturally, spiritually as well as psychologically with minimal or no external support at all (Quirk, R, 2001). Borrowing a leaf from an international economic order as coined by the Alma Ata Declaration of 1978 where it said that 'the rich and developed nations have the mandate to support the poor and developing nations' should not be take for granted. Should the support from rich and developed nations to the underdeveloped ones continue to make no positive impact on the nation in question then it becomes pathological, hence unacceptable. As such, the support needs not to continue but rather be revisited or another alternative should be sorted out.

In a family setting where children are raised up and groomed for adult life, an element of resilience such as self-dependence and ability to move on with life and form their own families is a normal and acceptable occurrence. Where the said children fail to move out from their parents' homes to start their own families or homes successfully and where parents continue to fully support the children during their adulthood, family life and homes, it is an indication that either the children or the parents have failed or something else is wrong. While the common saying goes "like begets like" or "like daughter, like mother and vice versa", the analogy here is that people must be responsible for their actions and live within their limits.

\subsection{Broad Objective}

To describe how to enhance the take-off of community based programmes, thereby improving livelihoods, self-dependence and sustainability. 


\subsection{Specific Objectives}

To describe how to enable -

1. Communities to identify programmes of their choice.

2. Communities to prioritize and own sustainable programmes.

3. Challenges of community based programmes to be identified and addressed logically and objectively.

4. Approaches to use to overcome obstacles preventing community based programmes from take-off.

\subsection{Community Involvement and Participation}

Why community do based programmes fail or succeed? The answer to this question is not all that simple as there are multiple factors. Individuals in the communities are not ignorant or stupid. They may not have gone to an elementary school or be unable to read and write but they are clever and knowledgeable of their perceived needs more than anybody else outside them can be. Mistakes are often made by the elite, upper class societies and those often called the owners of the market, the most literate, the rich or billionaires who prescribe what to do for the communities who are ill defined as marginalized. There are often unintentional grave mistakes made by development partners in the quest to support the communities. Planning and moving with the communities at every level of development is the key to succeed in as far as community based programmes are concerned. Therefore, community involvement and participation form the basis for the successful community based programmes and should never be overlooked in any way possible (Abrahamson and Abrahamson, 1999). Through this approach 'Community involvement and participation' communities are empowered to make informed choices, identify programmes of their choice based on their perceived needs, prioritize their needs and eventually own them. This will eventually facilitate ownership and sustainability which in essence prevent the well-established programmes from dying natural deaths.

\subsection{Intricacy of Community Involvement and Participation}

The two terminologies are so closely related to be inseparable since they complement or augment each other during the take-off of community programmes. Where one of them is overemphasized or underemphasized, then the course of action in question cannot take-off successfully.

3.2 Community involvement: this means taking part in an activity or event, emotionally, physically, socially, psychologically or otherwise and has a deeper meaning than the mere word 'participation'. In involvement, there is an element of a strong affinity for ownership or more experiential satisfaction without being pushed and where possible one is prepared to give their lives to it, such that they can die for the perceived cause of action if need be.

3.3 Community participation: ironically similar to involvement but in a more superficial way whereby there is an act of participating in an activity or event for the sake of it all but the actions can be withdrawn at any time without any strong feeling of pain, resentment or loss. However, combining the two terminologies when running a community based programme, it becomes a success and is the reason why they are used interchangeably and poses synergistic positive effects that are desirable to both parties (the implementer and the recipient).

3.4 An illustration of community involvement and participation: three colleagues namely; Mr. Pig, Mr. Chicken and Mr. Cow who were best friends had an event on a particular day and all of them needed to take part in the perceived event. It needed their community involvement and participation for the event -to be successful. The event in question was receiving a very important guest, long time best friend who had been away for over ten years. They were all excited and looking forward to having this friend at home but the assignment they had was to prepare him the best breakfast, an English breakfast for that matter. It took them the whole day to plan this task until one by one aired out their thoughts, commitments and contributions to the breakfast as stated below;

1. Mr. Pig: Yes, fresh potato crisps should be available but would be better to have pork sausages in place for him so as to make him enjoy the breakfast. Unfortunately, I do not have the money to buy sausages but I am prepared to be slaughtered and use my carcass for the sausages and this will have to be done without fail.

2. Mr. Chicken: Sure, I saw him a long time ago, so bread and tea would be ideal for him. However, breakfast without scrambled eggs would be incomplete. Therefore, as a Chicken, I am happily prepared to provide him with eggs for the breakfast and this will have to be done without fail.

3. Mr. Cow: 'Oh no, butter will be there, bread, tomatoes and everything you name, will still be there but tea without milk will make him uncomfortable and so, I will supply milk for the breakfast without fail'.

In no time, the guest arrived and there was excitement in the home as the surrounding was so tidy and welcoming. It was quite busy for each one of them to do their best and meet their promises. Eggs and milk were provided and it was the turn of Mr. Pig to provide sausages. Alas, the butcher had already been notified and arrived at the scene with his sharp knives, ready to slash Mr. Pig's throat in the quest for meat for sausages. Surprisingly, it was Mr. Pig himself who invited the butcher and made everything ready for the killing. Eventually. Mr. Pig was killed and his meat was prepared and provided as pork sausages for the guest's breakfast. The guest therefore had an English breakfast which consisted of among, other ingredients, pork sausages, scrambled eggs and tea with milk as the colleagues had agreed and provided.

Now comes the question regarding community involvement and participation. Of the three colleagues who fully got involved and participated in the event, an activity of providing an important guest with an English breakfast as per their initial arrangement? It is obvious that only Mr. Pig satisfied the full take off of the activity and therefore he got involved and satisfied in the true senses of the terminologies 'Community involvement and participation'. This was because Mr. Pig was prepared to die and indeed he died in order for the activity to be successful and sustainable. This is an attribute that has to be applied, utilized and implemented for community based programmes to be sustainable and successful which will lead to attainment of 'Acceptance and hope for achievement (AHA) Syndrome'.

\subsection{Acceptance and Hope for Achievement (AHA) Syndrome}

The AHA Syndrome came into scene in early 2000. Having been tried in over 10 community based programmes, it was substantiated and evidenced as key resilience for sustainable community based programmes. This is because the community based programmes that applied this AHA Syndrome flourished and were sustained. This write up is therefore meant to spice up and share AHA Syndrome with the rest of the international and local development partners who are prepared to make long positive and meaningful transformation of community based programmes to benefit the marginalized and ultra-poor communities worldwide. Below is the explanation of AHA Syndrome.

4.1 Acceptance: In the first place, partners of good will, willing to implement long lasting community based programmes must be willing to involve the local communities at grass root levels right from the word go. At every stage of development, such as planning, communities have to be involved, be it identifying the community needs, prioritizing, setting objectives, mapping, problem statement, data collection, analyzing the 
findings, implementing the findings and application of the findings. The communities need to be supported to initiate the programme of their choice and be able to reach the full level of 'Acceptance'. When the communities come to a consensus or realization and say, 'yes, this is what we need', it is an indication that the first level of initiating a sustainable community based programme has begun. This means now that they are able to envisage or see the fruits, hence the benefits. This moves us to the following second level.

4.2 Hope: at this level, a sense of hope has been created in the minds of opinion leaders at all levels; women, men and youths are now nurturing the future in anticipation positive change and there is confidence in themselves that they are capable of doing something meaningful. The communities are able to envisage the big picture in their own way as true agents of change, though in abstract form. They strive to translate their thoughts from cognitive processes to concrete perspectives. This atmosphere creates a sense of ownership and self-reliance as well as selfdependency, and moves on to the third level.

4.3 Achievement: at this level, communities see themselves as true change agents at best and ready to implement their thoughts and see the fruit of their labor. When they witness change in the positive direction, for instance where malnutrition programme is being implemented and see the number of malnourished children getting reduced, this especially gives them satisfaction, pride and excitement in the fruits of their labor. It is at this level when communities say ' AHA, this is our programme' as opposed to 'it is their programme'. Here the community based programme has taken-off and the probability of sustainability is relatively high. Therefore, the combination of the aforementioned three levels is what is termed as 'Acceptance and Hope for Achievement (AHA) Syndrome', as key to resilience for community based programmes.

\subsection{Limitations}

In any given task, there are squabbles that are faced and in most cases these are normal occurrences. Where possible such bottle necks should be used as stepping stones for moving forward hence cherished when they are met. In this write up, pragmatically time constraint has certainly been one of the challenges faced during the process. However, the paper is a straightforward guide and so has the strength of informing potential programme financiers, policy makers, strategic planners and implementers when coming up with successful and sustainable community based programmes that would benefit the deserved communities worldwide.

\subsection{Conclusions and Recommendations}

Community based programmes can be viewed as tools for community development. Different approaches are used to make the programmes successful but often it proves futile after millions of dollars have been spent and eventually leave the communities poorer than they were before the programmes. It is for this reason that careful determination of the programmes and communities in question need to be considered and become the main stay, making sure that at every step of development communities are involved. Helping the communities to make informed choices and reach an acceptance level facilitates the sustainability of such community based programmes. As multiple tools are applied to enhance community based programmes take-off, so one of such tools is the 'Acceptance and hope for Achievement (AHA) Syndrome'. The authors recommend that the proven AHA Syndrome be implemented to effect meaningful change in the diverse communities that are intended to be supported and transformed from poverty to prosperity. Where necessary, authors are willing to be contacted to give practical advice about planned community based programmes as 'Together we can make the difference'.

\section{Acknowledgements}

It takes more than one person to come up with the piece of work of this nature. However, I would like to thank my daughter Lettice and the rest of my family members for their encouragement to have this work shared when I narrated the idea with them. Similarly, appreciation goes to my colleagues at work for the encouragement ever since I planned to have this piece of work published to share with the rest of the world communities. Further, thanks to Professors Peter Byass, Anna-Karin, Heather Roberts, Maria Emmelin, Roy Shafer, Gilbert Burnham and others too numerous to mention for their valuable coaching and introduction to the world of Public Health. Additional thanks go to my long time and family friends Kath and John Young and Ludger Heuckmann for their valuable support in kind.

Finally though not least, I would like to thank deeply the communities that I have worked with all the years of cherishing this concept, glad that it will touch different lives across diverse cultures, communities worldwide.

\section{References}

\section{Alma Ata Declaration of 1978, USSR.}

2. Randolph, Quirk. (2001). Longman Dictionary of Contemporary English, Third edition with New Words supplement.

3. Abrahamson, J.H., Abrahamson ZH (1999). Survey Methods in Community Medicine, Epidemiological, Research Programme Evaluation, Clinical Trials, Fifth edition, Churchill Livingstone, Edinburgh London New York, Philadelphia, Sydney, Toronto. 